\title{
EXPERIMENTAL DETERMINATION AND THEORETICAL ANALYSIS OF LOCAL RESIDUAL STRESS AT GRAIN SCALE
}

\author{
INDRANIL BASU, VÁCLAV OCELÍK \& JEFF TH. M. DE HOSSON \\ Department of Applied Physics, University of Groningen, The Netherlands
}

\begin{abstract}
Grain/phase boundaries contribute significantly to build up of residual stresses, owing to varied plastic/thermal response of different grain orientations or phases during thermomechanical treatment. Hence, accurate quantification of such local scale stress gradients in commercial components is important in understanding their mechanical performance. The current work introduces a correlative method utilizing Electron Back Scattered Diffraction and Focused Ion Beam - Digital Image slit milling methodology to accurately determine spatially resolved stress profiles in the vicinity of grain boundaries using commercially pure titanium as a model material. Measured local stress gradients were in good agreement with local misorientation values. The role of dislocation-grain boundary interactions on buildup of local stress gradients is elucidated. Stress profiles near grain boundaries initially display non Hall-Petch characteristics, followed by a typical Hall-Petch type variation of "one over square root of distance". The observed trends allude to local stress relaxation mechanisms very close to the grain boundaries. The findings indicate that grain scale stress gradients can be significant in terms of playing a crucial role in macroscopic fatigue behavior.

Keywords: electron back scattered diffraction, focused ion beam milling, grain boundary damage, fatigue, internal stresses, digital image correlation.
\end{abstract}

\section{INTRODUCTION}

The term "residual stress" prescribes to locked in stresses, which remain after materials processing. These stresses span across different length scales. The influence of microstructure, i.e. spatial distribution of grains and phases, is significant on local residual stress distribution. In particular, grain boundaries contribute significantly to build up of such internal stresses, owing to varied mechanical response of different grain orientations during plastic deformation.

In metals, the motion and interaction of dislocations within the material microstructure and their resultant impact on micro scale plasticity is crucial for understanding the macroscopic mechanical performance and failure resistance of a component. Dislocations can not only interact among themselves but also with other crystal defects such as grain boundaries, often giving rise to complex geometrical configurations of stored dislocations that are associated with long-range elastic stress fields. Superposition of such stress fields invariably results in a strong spatial heterogeneity in local stress states, which directly determines preferential zones for damage nucleation and thereby fracture characteristics in materials.

Stored dislocations are generally classified into two types viz. geometrically necessary dislocations (GNDs) that accommodate a lattice curvature from a deformation gradient; and statistically stored dislocations (SSDs), which accumulate due to statistical entanglements. While SSDs are prone to rearrangement by thermally activated processes such as cross-slip or climb, GNDs maintain the lattice continuity across multiple grains during plastic deformation, hence acting as the primary contributors to strain hardening. Needless to say, the variation of local GND density levels directly influences the distribution of residual 
stresses in the interior of the grain. Such correlation between GND density levels and local stress gradients has been utilized in the past to explain local hardening phenomenon due to dislocation pile up at grain boundaries. Eshelby et al. [1], showed analytically that the dislocation pile up ahead an insurmountable obstacle such as grain boundary would result in a stress gradient that varies as 'one over square root' of the distance from the obstacle. Subsequent experimental observations by Hall [2], [3], and Petch [4], independently reestablished such a behavior in metals as the well-known Hall-Petch effect, wherein the mechanical strength of the material increases with decreasing grain size.

The dislocation configuration near a grain boundary strongly determines the degree of pile up and corresponding local stress concentration. Depending on the crystallography of the grain boundary certain slip systems may find conjugate systems in the neighboring grain that facilitate complete or partial slip transfer. A theoretical estimate of the feasibility of slip transmission can be described by the slip transfer parameter [5], [6], expressed as,

$$
m^{\prime}=\left(\boldsymbol{n}_{\mathbf{1}} \cdot \boldsymbol{n}_{\mathbf{2}}\right) \cdot\left(\boldsymbol{b}_{\mathbf{1}} \cdot \boldsymbol{b}_{\mathbf{2}}\right),
$$

where $\boldsymbol{n}_{\mathbf{1}}$ and $\boldsymbol{n}_{\mathbf{2}}$ are the normalized intersection lines common to the slip planes and the boundary plane, and $\boldsymbol{b}_{\mathbf{1}}$ and $\boldsymbol{b}_{\mathbf{2}}$ are the normalized slip directions in the pile-up and emission grains. The value of $m^{\prime}$ provides a measure of the probability for possible transmissivity of a dislocation across the grain boundary. Maximization of slip transfer parameter $m^{\prime}$ abates dislocation pile up and promotes easier slip transfer across the grain boundary.

Quantitative measurement of spatially dependent local stress states near grain boundaries therefore becomes crucial in understanding fracture mechanisms in structural materials. Spatially resolved internal stress measurements can be made using high resolution electron back scatter diffraction (HR-EBSD) wherein Kikuchi patterns from reference (un-deformed) and deformed states are cross-correlated to measure the residual elastic strains, and subsequently calculate the local elastic stress state [7], [8]. However, the method is limited to 2-dimensional investigations wherein only surface information is obtained [9]. With the advent of dual-beam focused ion beam (FIB) - field emission gun microscopes, measurement of residual stresses with simultaneous sub-micron lateral and depth resolution in a semi-automated and robust way [10], is made possible. In this way both surface and bulk deformation contribution on internal stress build up is accounted during stress quantification. The methodology utilizes correlative imaging and milling to remove material and estimate the local stress relaxation in the neighborhood. Digital image correlation (DIC) is utilized to determine relaxation induced displacements in the vicinity of the milled region.

The novelty of the current work lies in introducing a site specific method utilizing electron back scattered diffraction (EBSD) and FIB-DIC slit milling to accurately determine spatially resolved stress profiles in the vicinity of grain boundaries in commercially pure titanium. Correlations with the GND dislocation density and slip transfer parameter are drawn to validate the stress measurements. The observed trends are subsequently discussed with respect to underlying physical processes and its subsequent impact on fracture resistance of titanium is acknowledged.

\section{EXPERIMENTAL METHODS}

Commercially available grade II titanium was subjected to room temperature in-situ four-point bending test inside a Tescan Lyra dual beam (FEG-SEM/FIB) scanning electron microscope. Prior to mechanical testing, bending specimens were prepared for EBSD measurements using conventional metallographic techniques [11]. Specimens were strained to a final surface true strain, $\varepsilon=0.05$. Microstructural characterization was performed by means of EBSD, thereby extracting both topographical and orientation information of 
individual grains. A step size of $0.3 \mu \mathrm{m}$ and hexagonal type of grid was used for the measurements. The acquired raw EBSD data was subsequently analyzed using EDAX-TSL OIM $^{\mathrm{TM}}$ Analysis 7.3 software and MTEX Matlab toolbox [12]. Slip traces in individual grains were imaged using in-situ scanning electron microscopy (SEM). The orientation of the grain boundary plane was determined by milling into the region containing the boundary using focused ion beam and examining the grain boundary trace along the milled cross section. All observations were made on the tensile surface of the bent specimen, with the direction of viewing parallel to the surface normal, hereinafter referred to as A3 sample axis.

\subsection{Residual stress measurement by FIB-DIC slit milling}

The protocol followed during the measurement of residual stress starts with the acquisition of a scanning electron microscopy (SEM) image of the area to be analyzed. After recording the first image, a slit is milled on the surface. Then, a second image of the same area is taken. From the comparison of these two SEM images recorded before and after stress release by DIC the displacements are obtained. These displacements are compared with those obtained by the analytical solution for an isotropic elastic material, and the value of residual stress is obtained from the slope of the fitting.

Fig. 1 shows the geometry of the slit used in our experiments including its dimensions: a length $L$, a width $w$ and a depth $a$. The evaluated displacements of the surface, $u_{x}$, are normal to the plane of the slit. The origin of coordinates is placed at the center of the slit. Considering the geometry, in plane displacements $U_{d i r}$ can be related to residual stress $\sigma_{d i r}$ in the same direction by an analytical expression (see eqn (1)) such as:

$$
U_{\text {dir }}=\frac{2.243}{E^{\prime}} \sigma_{\text {dir }} \int_{0}^{a} \cos \theta\left(1+\frac{\sin ^{2} \theta}{2(1-v)}\right) \times(1.12+0.18 \cdot \operatorname{sech}(\tan \theta)) d z,
$$

where $E^{\prime}$ is $E /\left(1-v^{2}\right), E$ is the Young's modulus, $v$ is the Poisson's ratio, $\theta$ is $\arctan (d / a), a$ is the depth of the slit and $d$ is the distance from the slit; 'dir' represents $\mathrm{x}$ or $\mathrm{y}$ directions. The displacements caused by the stress release depend on the slit depth $a$ and are directly proportional to $\sigma / E$ ratio. Moreover, the extraction of the residual stress value requires the previous knowledge of the elastic properties of the material under study (i.e. Young's modulus and Poisson's ratio).

SEM images acquired before and after FIB milling are processed using a commercial digital image correlation software GOM Correlate v. 2016 and subsequent displacements lateral to the slit are recorded for each facet (group of pixels) position. Fig. 2 represents the contour map of relative displacements normal to the slit corresponding to the local stress release due to milling. The scale bar of the image is in the range of tens of nanometers.

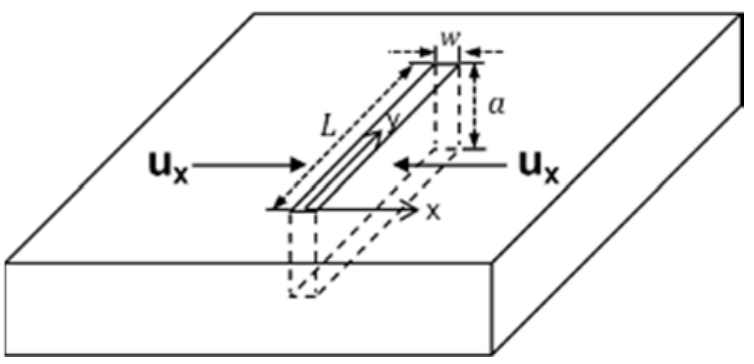

Figure 1: Schematic of slit introduced by FIB milling. 
Each color means that a group of pixels is displaced over the respective number of nanometers. When studying the displacements normal to the plane of the slit, the displacement is to the right (red color) or to the left (blue). Consequently, data arrays comprising of facet id, coordinates and relative displacements are exported for post processing and residual stress determination. A Matlab based script is utilized to empirically determine the residual stress values from the experimentally measured displacements, as per eqn (2).

In the current work, linear slits, oriented normal to the grain boundary trace, of a fixed width $0.5 \mu \mathrm{m}$, depth $2.5 \mu \mathrm{m}$, and lengths varying from 15-25 $\mu \mathrm{m}$ (depending on the grain size), were milled inside individual grains showing pile up as per local misorientation data. Measurements were performed on multiple grains showing different degrees of pile up. For each slit, multiple SEM images of resolution $768 \times 768$ pixels were acquired at high magnifications (field of view of 10-15 $\mu \mathrm{m}$ ) to ensure a high spatial resolution of measured displacement field. In order to obtain statistically sufficient data points, DIC was performed using a facet size of $19 \times 19$ pixels with a step width of 16 pixels. Yttria-stabilized Zirconia (YSZ) nano-particles were used for surface decoration to obtain optimum image contrast for high accuracy DIC analysis.

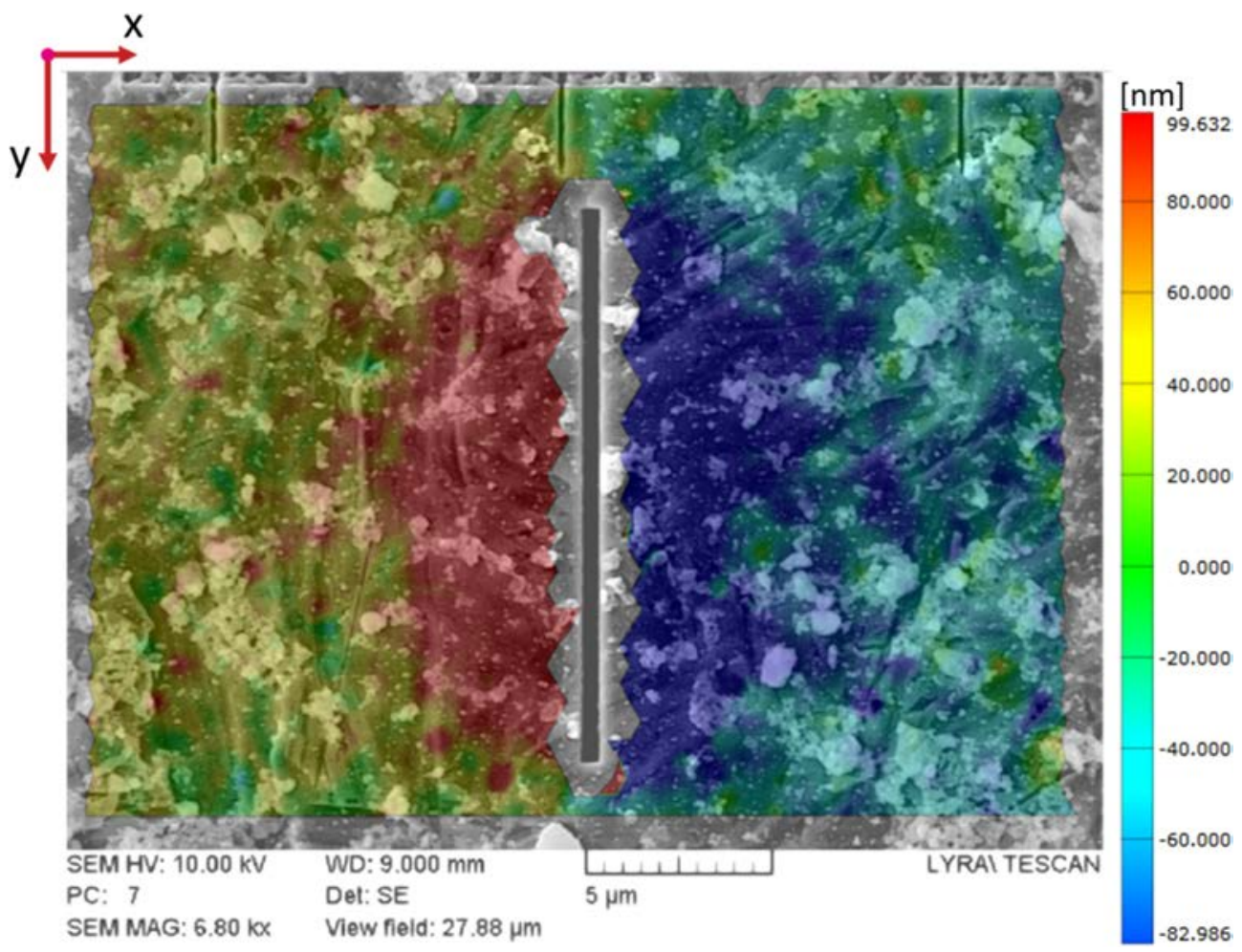

Figure 2: Displacement field map from DIC analysis in GOM. 

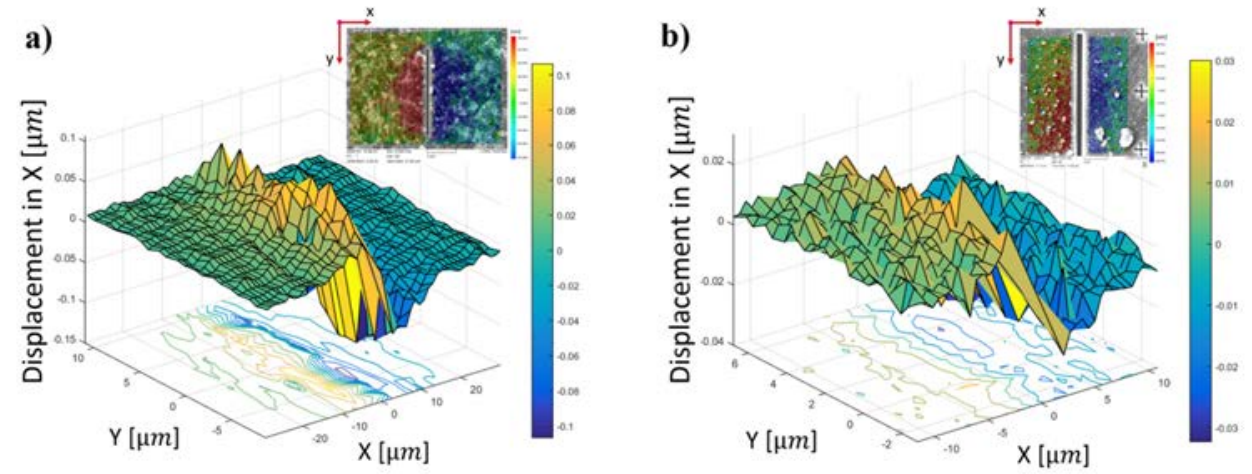

Figure 3: Displacement contour maps illustrating (a) Homogeneous; and (b) Heterogeneous displacements fields.

a) Singular fitting

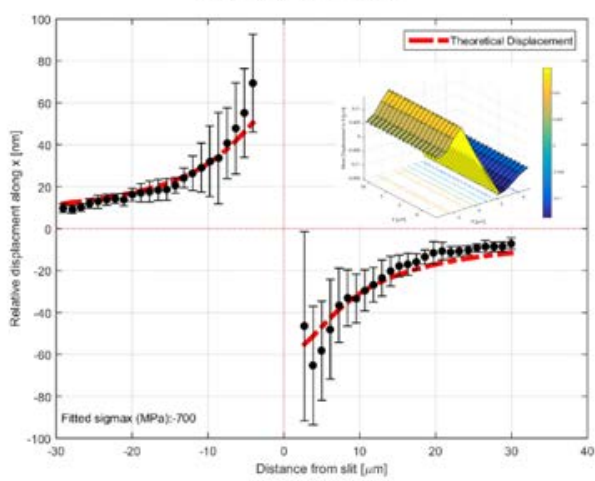

b) Multiple fitting

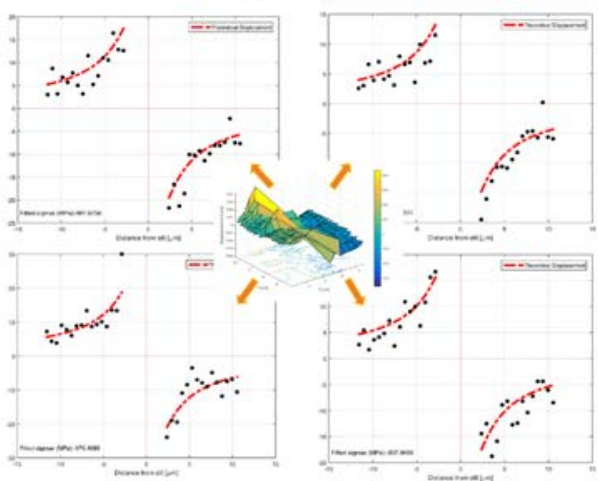

Figure 4: (a) Singular fitting approach - Displacement are averaged along the slit and plotted as a function of lateral distance from the slit. Empirical fitting to theoretically calculated displacements as described in ref [13], gives the average local residual stress corresponding to the slit; (b) Multiple fitting approach - Individual fitting of displacements for each row performed over all the rows along the length of the slit, stress value obtained for each row of displacement.

Stress distribution at sub-microscopic length scales need not always be homogeneous i.e. constant stress all throughout the material, but may considerably vary spatially. Fig. 3(a) and Fig. 3(b) illustrate examples of homogeneous and heterogeneous displacements. In such cases, stress determination by simplistic averaging of all displacements along the slit length i.e. singular fitting (cf. Fig. 4(a)), misrepresents the actual stress state of the material. A multiple fitting approach [13], wherein a stress value is obtained for displacements corresponding to each row (cf. Fig. 4(b)), was implemented to account for spatially heterogeneous stress states. Fig. 5 shows the corresponding deviation between the stress values measured by multiple fitting approach in comparison with that obtained from singular fitting (one stress value for the whole slit). In order to simplify calculations, an isotropic 
elastic modulus tensor was assumed for stress determination from the measured displacements.

\section{RESULTS AND DISCUSSION}

Fig. 6 illustrates a representative case of a grain associated with a strong pile up, labelled as Grain 2. Fig. 6(a), Fig. 6(b) and Fig. 6(c) represent the Kernel Average Misorientation (KAM), Local Average Misorientation (LAM) and the grain boundary slip transmissivity $\left(m^{\prime}\right)$ maps respectively. The KAM physically describes the average misorientation spread between a reference pixel and its nearest neighbor pixels for a defined kernel size. The LAM angle corresponds to the misorientation averaged over all nearest neighbor pairs within a kernel. Both LAM and KAM values were calculated for the $2^{\text {nd }}$ nearest neighbor with a threshold value of $2^{\circ}$ [14]. High angle grain boundaries, classified as larger than $15^{\circ}$, are highlighted in white in Fig. 6(a) and Fig. 6(b). The KAM and LAM maps indicate a strong pile up at the grain boundary separating Grain 1 and Grain 2. The grain orientations in Fig. 6(c) are depicted as unit cells. The viewing axis corresponds to the $A 3$ direction. The grain boundaries in Fig. 6(c) are additionally colored with respect to their respective slip transfer parameter value that ranges from dark blue corresponding to no slip transfer to light yellow indicating complete transmissivity. The grain boundary segment between Grain 1 and Grain 2 displays a low probability of slip transfer, with an $m^{\prime}$ value between 0 and 0.1 . Fig. $6(d)$ shows the variation of local average misorientation (LAM) and GND density with respect to distance from the grain boundary (refer to highlighted line AB in Fig. 6(a)).

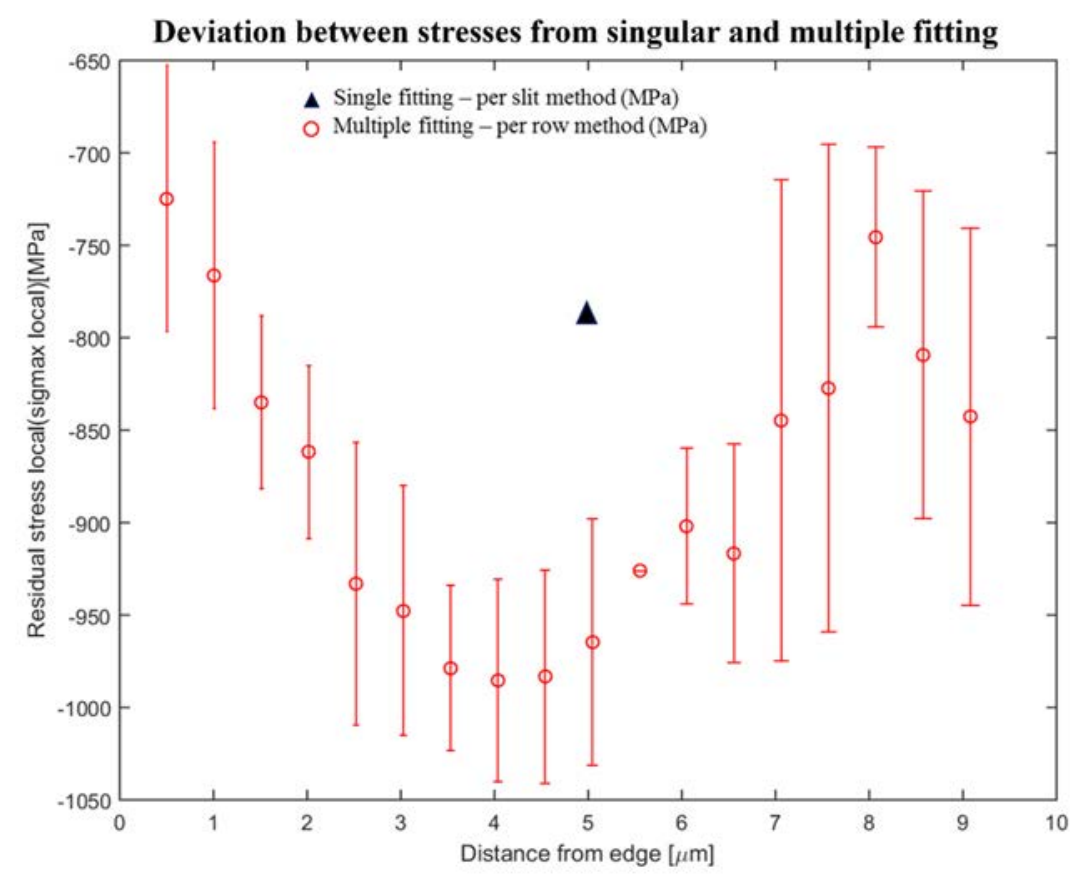

Figure 5: Deviation in stress values from multiple fitting approach vis-à-vis singular fitting method. 
a)
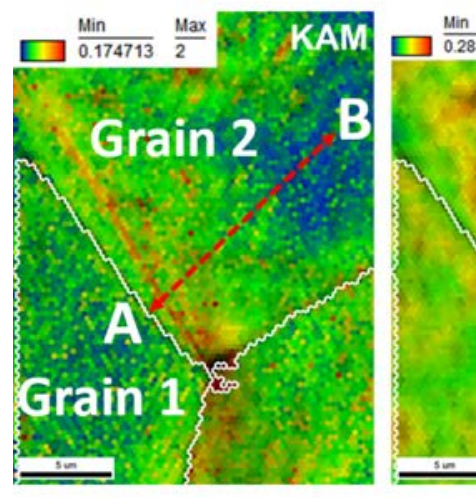

b)

$\frac{\max }{0.874635}$

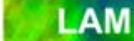

LAM

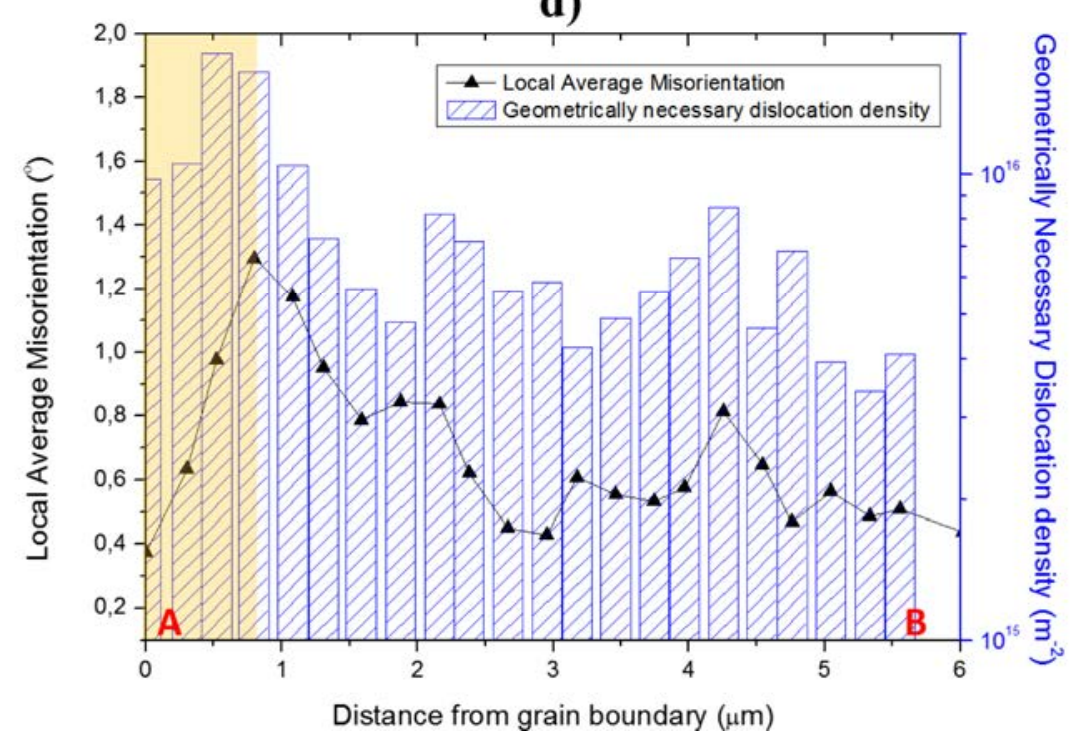

c)

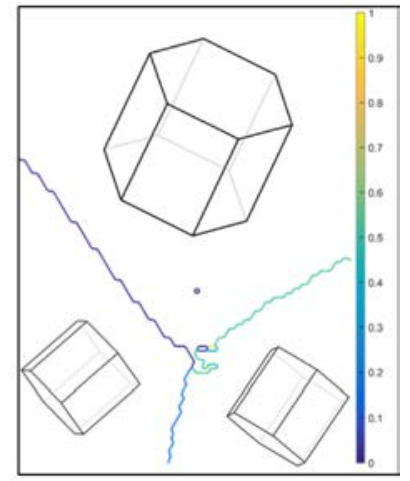

d)

Figure 6: (a) KAM; (b) LAM; and (c) m' maps for Grain 2 and neighborhood; (d) GND density and LAM profile between points A and B.

GND density $\left(\rho_{G N D}\right)$ values from EBSD data were calculated using the strain gradient approach [15], [16], given by the expression:

$$
\rho_{G N D}=\frac{2 \theta}{n a\left|\boldsymbol{b}_{\boldsymbol{d}}\right|},
$$

where $\theta$ is the experimentally measured KAM value, $a$ is the step size, $n$ is the number of nearest neighbors averaged in the KAM calculation and $\boldsymbol{b}_{\boldsymbol{d}}$ is the Burgers vector corresponding to the active slip system in the grain. The excellent agreement between the LAM and GND values is unsurprising since both values are derived from local misorientation. Interestingly both trends reveal a local minimum close to the grain boundary highlighted by the shaded region in Fig. 6(d). 
a)

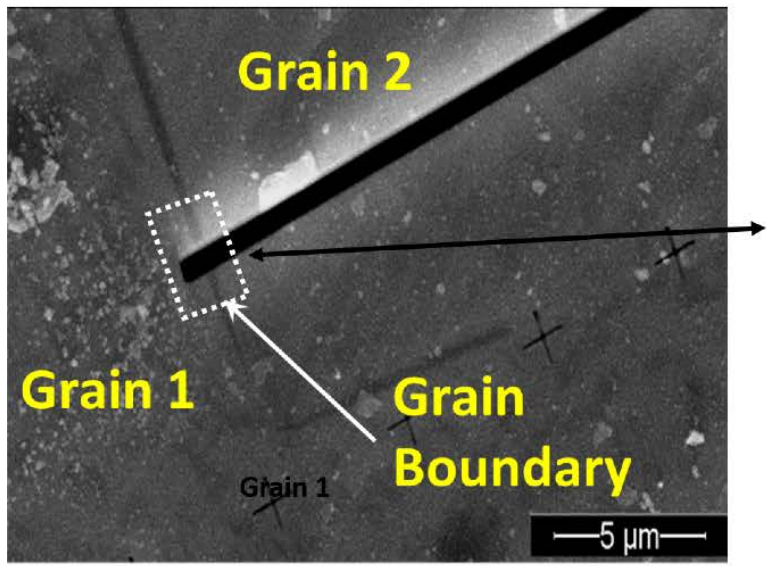

b)

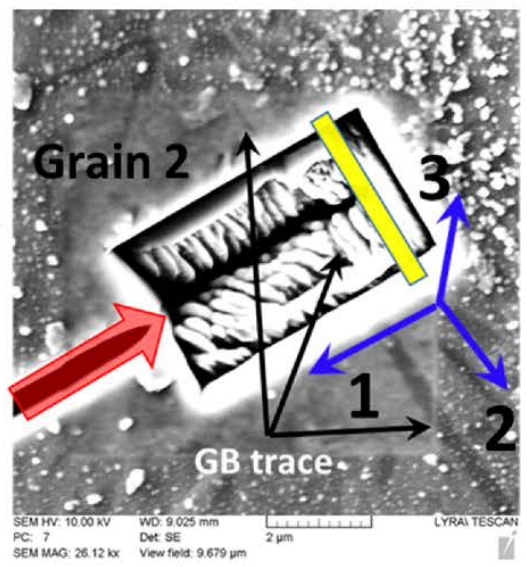

c)

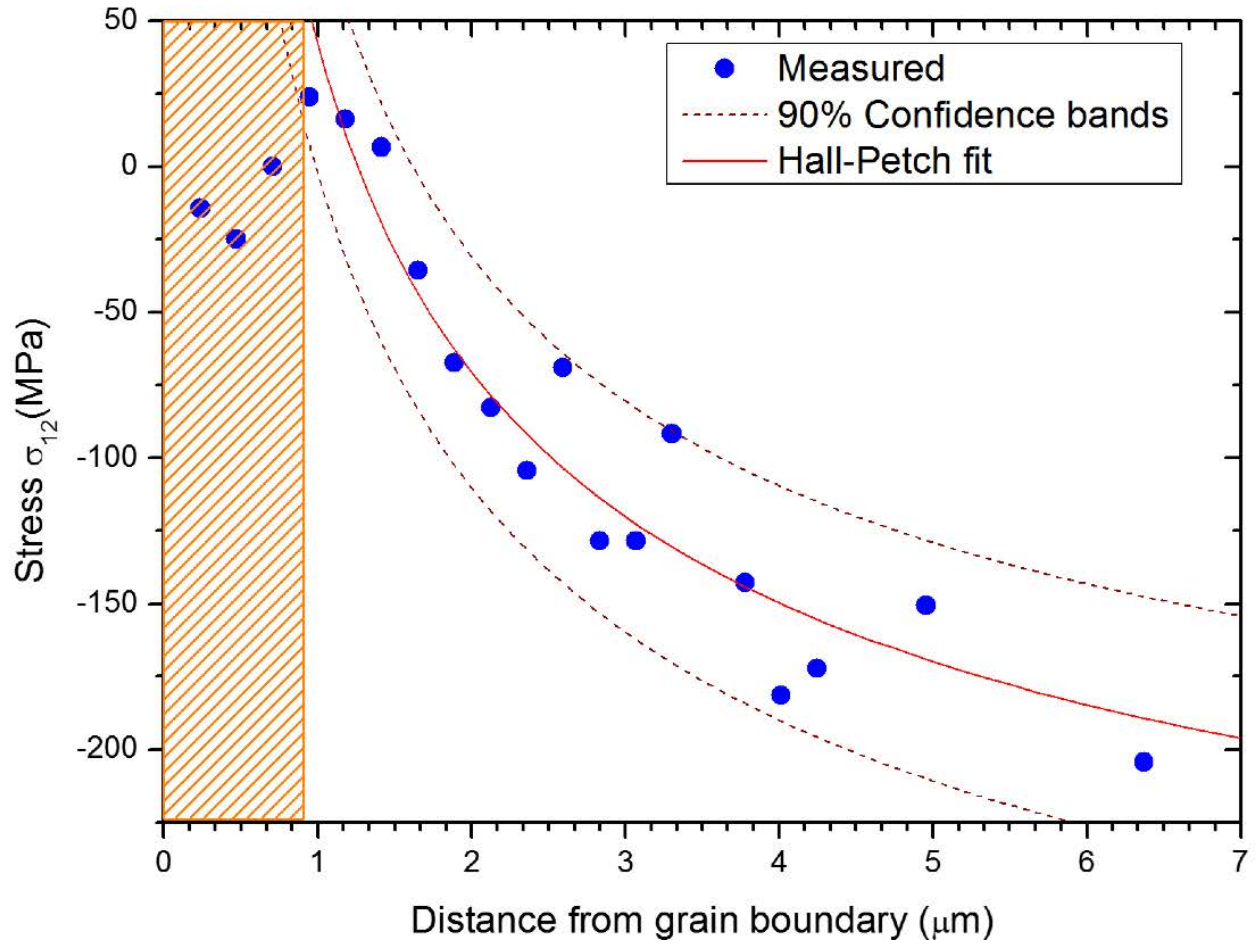

Figure 7: (a) SEM image showing slit; and (b) grain boundary plane orientation (red arrow indicates longitudinal direction of the slit); (c) corresponding measured stress profile between points $\mathrm{A}$ and $\mathrm{B}$. 
Fig. 7 represents the FIB-DIC analysis and corresponding stress measurements for the region shown in Fig. 6. Fig. 7(a) shows the spatial orientation of the milled slit with respect to the grain boundary. The white dots correspond to the YSZ particles used for surface decoration. Grain 2 shows profuse slip bands that were identified as (10 $\overline{1} 0)$ prismatic plane traces. Fig. 7(b) represents the orientation of the grain boundary plane with respect to the slit direction represented by the red arrow, indicating that longitudinal direction of slit is parallel to the grain boundary normal within the measurement volume. Fig. 7(c) displays the measured stress profile as a function of normal distance from the grain boundary. The measured stress component $\sigma_{12}$ describes the stress acting parallel to the grain boundary plane and perpendicular to the length of the slit. The stress values within a distance of 0.9 $\mu m$ from the grain boundary show a local minimum, which is succeeded by a stress peak that monotonically decreases with increasing distance from the grain boundary. On comparing the stress gradient with Fig. 6(d) the agreement seems excellent, thereby indicating that the observed stress fluctuations indeed confer to the actual stress state near the grain boundary. The data points corresponding to distances greater than $0.9 \mu \mathrm{m}$ show a good fit to the theoretically known 'one over square root of distance' Hall-Petch variation [2]-[4], (cf. Fig. 7(c)). It is worthwhile to note that the stress values fluctuate between compressive and tensile characteristics near the grain boundaries with gradual transition into compressive stresses in the grain interior. The Hall-Petch coefficient $k_{H P}$ corresponding to Grain 2 is empirically determined to be $0.381 \mathrm{MPa}$.m 0.5 .

The initial stress minimum observed in Fig. 7(c) indicates activation of local stress relaxation mechanisms, wherein dislocation spacing seems to equilibrate in regions very close to the boundary i.e. pile up effect is abated locally. Slip trace analysis inside Grain 2 revealed activation of more than one prismatic slip systems near the grain boundary (most likely nucleating at the grain boundary due to stress fields exerted by the neighboring grain), whereas singular slip took place in the grain interior (cf. Fig. 7(a) and Fig. 8(a)). Activation of multiple deformation modes would locally enhance dislocation - dislocation interactions close to the boundary i.e. promote passing and cutting stresses, which can significantly retard dislocation motion in the region.

a)

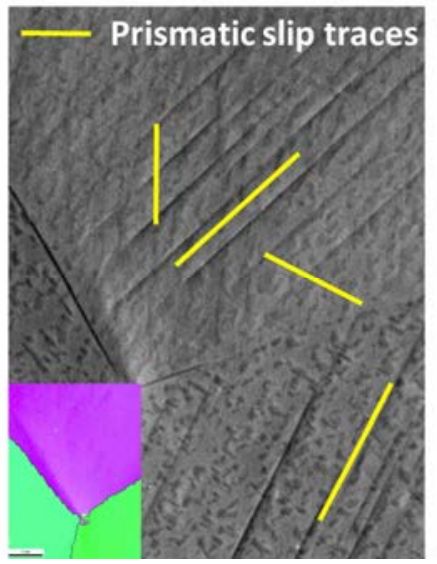

b)

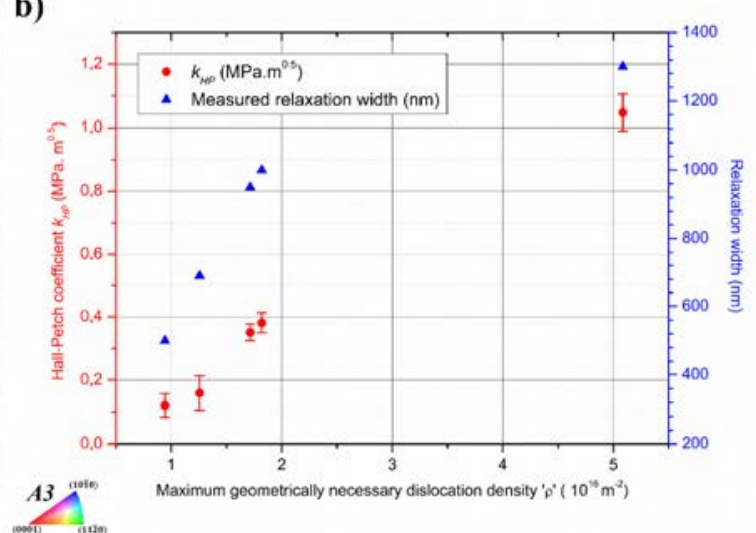

Figure 8: (a) SEM image of prismatic slip traces, inverse pole figure map depicting color gradient due to slip induced lattice rotation near the grain boundary; (b) variation of $k_{H P}$ and relaxation width with the peak GND density measured in different grains. 
Another contributing factor could be the change in the local stress state very close to the grain boundary that may lead to spatial delocalization of dislocation cores at the tip of the pile up, thereby locally depleting the dislocation density ahead of the pile up. During plastic deformation, lattice dislocation segments trapped by the grain boundary may overlap with dislocation cores of grain boundary dislocations (GBDs), giving rise to non-equilibrium grain boundary configurations. The resultant elastic stress fields generated from such interactions are long ranged in nature and can become sufficiently high to influence dislocation slip in a rather thick grain boundary mantle [17]-[19]. The observed spread in the KAM values (cf. Fig. 6(a)) along the grain boundary for Grain 2 most likely results from such local spreading of dislocations at the tip of pile ups, originating from multiple slip-grain boundary intersection sites.

Since the local residual stresses determined post plastic deformation are directly linked to the magnitude of elastic stresses exerted by the (edge) dislocations present in that region, the observed stress relaxation near the grain boundary is attributed to relative decrement in the dislocation density levels at the grain boundary. Fig. 8(b) shows the variation of Hall-Petch coefficient $k_{H P}$ and the width of the relaxed volume as a function of the peak value of GND density for different grains. The results indicate that both the width of volume experiencing stress relaxation in a grain as well as the Hall-Petch coefficient $k_{H P}$ increases with higher GND density levels. The trends also reveal that for higher $k_{H P}$ values the width of the zone near the grain boundary showing stress minimum increases. The Hall-Petch coefficient, also called the dislocation locking parameter, typically describes the resistance to slip nucleation or transfer in the neighboring grain [20]. In other words, larger the value of $k_{H P}$, more and more lattice dislocation segments will be absorbed by the grain boundary i.e. higher trapped lattice dislocation (TLDs) densities. Since the elastic stress field from the interaction between

TLDs and GBDs varies linearly with $\sqrt{\rho_{T L D}\left(m^{-1}\right)}$, higher TLD densities $\left(\rho_{T L D}\right)$ would subsequently lead to aforementioned delocalization effects over a wider zone adjacent to the grain boundary [19]. For instance, assuming a $\rho_{T L D}$ in the range of $10^{8} \mathrm{~m}^{-1}$, non-equilibrium grain boundary stress fields can be of the order of $10^{-3} G$ even at distances $\approx 10^{4} . b_{d}$ (where, $G$ is the shear modulus and $b_{d}$ is the Burgers vector for active dislocation slip) from the grain boundary plane. Also noteworthy is the significant scatter seen in the $k_{H P}$ values for different grains (cf. Fig. 8(b)), compared to the average Hall-Petch coefficient for polycrystal Ti of $0.4 M P a . m^{0.5}$ [21]. The large fluctuations in Hall-Petch coefficients suggest its strong dependence upon the local grain orientation and grain boundary geometry.

The implications of the observations in the current work are significant in terms of understanding fracture resistance in titanium. A tensile stress state at the grain boundary would promote fracture by crack nucleation [22], and hence highlights the detrimental role of grain boundaries in fracture resistance. However, the measured stress profile in Fig. 7(c) indicates a local relaxation close to the boundary wherein the local stresses transition from tensile to compressive nature on moving towards the grain boundary. These observations shed light upon locally active intrinsic mechanisms that may aid in delaying damage nucleation at grain boundaries.

\section{CONCLUSIONS}

A novel correlative technique utilizing EBSD and FIB-DIC method for obtaining site specific microstructural and local stress information is presented. Stress gradients due to dislocation pile up at pure titanium grain boundaries are quantified. The following conclusions are drawn: 
1. Stored dislocation densities and stress values near the grain boundary revealed a local minimum, deviating from the expected Hall-Petch characteristics.

2. The observed stress drop is justified by a local change in elastic stress fields arising from dislocation-dislocation and dislocation-grain boundary interactions that may lead to a relative depletion of dislocation densities in the vicinity of specific grain boundaries.

3. The $k_{H P}$ values measured for different grains ranged from $0.14 \mathrm{MPa} . \mathrm{m}^{0.5}$ to 1.047 MPa. $\mathrm{m}^{0.5}$, indicating a strong dependence of the local strengthening behavior on the grain orientation and grain boundary crystallography. The width of the zone undergoing stress relaxation near a grain boundary scales non-linearly with increasing value of $k_{H P}$ and peak dislocation densities, varying from $500 \mathrm{~nm}$ to $1300 \mathrm{~nm}$.

4. The observations in the current work have significant implications on the generic understanding of grain boundary damage mechanisms.

\section{ACKNOWLEDGEMENTS}

This research was carried out under project number T61.1.14545 in the framework of the research program of the materials innovation institute (M2i) (www.m2i.nl). Indranil Basu would like to thank Herman Fidder for providing titanium material.

\section{REFERENCES}

[1] Eshelby, J.D., Frank, F.C. \& Nabarro, F.R.N., XLI. The equilibrium of linear arrays of dislocations. Lond. Edinb. Dublin Philos. Mag. J. Sci., 42(327), 351-364 (1951).

[2] Hall, E.O., The deformation and ageing of mild steel: III Discussion of results. Proc. Phys. Soc. Sect. B, 64(9), pp. 747-752, 1951.

[3] Hall, E.O., Variation of hardness of metals with grain size. Nature, 173, pp. 948-949, 1954.

[4] Petch, N.J., The cleavage strength of polycrystals. J Iron Steel Inst, 174, pp. 25-28, 1953.

[5] Shen, Z., Wagoner, R.H. \& Clark, W.A.T., Dislocation and grain boundary interactions in metals. Acta Metall, 36(12), pp. 3231-3242, 1988.

[6] Clark, W.A.T. et al., On the criteria for slip transmission across interfaces in polycrystals. Scr. Metall. Mater, 26(2), pp. 203-206, 1992.

[7] Britton, T.B. \& Wilkinson, A.J., Measurement of residual elastic strain and lattice rotations with high resolution electron backscatter diffraction. Ultramicroscopy, 111(8), pp. 1395-1404, 2011.

[8] Britton, T.B. \& Wilkinson, A.J., Stress fields and geometrically necessary dislocation density distributions near the head of a blocked slip band. Acta Mater., 60(16), pp. 5773-5782, 2012.

[9] Guo, Y. et al., Measurements of stress fields near a grain boundary: Exploring blocked arrays of dislocations in 3D. Acta Mater., 96(1), pp. 229-236, 2015.

[10] Winiarski, B. \& Withers, P.J., Novel implementations of relaxation methods for measuring residual stresses at the micron scale. J. Strain Anal. Eng. Des., 50(7), pp. 412-425, 2015.

[11] Taylor, B. \& Weidmann, E., Metallographic preparation of titanium, struers application notes. Struers Den. Rosendahls Bogtryk, 2008.

[12] Hielscher, R. \& Schaeben, H., A novel pole figure inversion method: specification of the MTEX algorithm. J. Appl. Crystallogr, 41, pp. 1024-1037, 2008. 
[13] Mansilla, C., Martínez-Martínez, D., Ocelík, V. \& De Hosson, J.T.M., On the determination of local residual stress gradients by the slit milling method. J. Mater. Sci, 50, pp. 3646-3655, 2015.

[14] Calcagnotto, M., Ponge, D., Demir, E. \& Raabe, D., Orientation gradients and geometrically necessary dislocations in ultrafine grained dual-phase steels studied by 2D and 3D EBSD. Mater. Sci. Eng A, 527(10-11), pp. 2738-2746, 2010.

[15] Kubin, L.P. \& Mortensen, A., Geometrically necessary dislocations and strain-gradient plasticity: a few critical issues. Scr. Mater, 48(2), pp. 119-125, 2003.

[16] Konijnenberg, P.J., Zaefferer, S. \& Raabe, D., Assessment of geometrically necessary dislocation levels derived by 3D EBSD. Acta Mater, 99, pp. 402-414, 2015.

[17] Grabski, M.W. \& Korski, R., Grain boundaries as sinks for dislocations. Philos. Mag., 22(178), pp. 707-715, 1970.

[18] Pond, R.C. \& Smith, D.A., On the absorption of dislocations by grain boundaries. Philos. Mag, 36(2), pp. 353-366, 1977.

[19] Nazarov, A.A., Romanov, A.E. \& Valiev, R.Z., On the structure, stress fields and energy of nonequilibrium grain boundaries. Acta Metall. Mater, 41(4), pp. 1033-1040, 1993.

[20] Armstrong, R., Codd, I., Douthwaite, R.M. \& Petch, N.J., The plastic deformation of polycrystalline aggregates. Philos. Mag., 7(73), pp. 45-58, 1962.

[21] Gottstein, G., Physical Foundations of Materials Science. Springer Science \& Business Media, 2013.

[22] Irwin, G., Analysis of stresses and strains near the end of a crack traversing a plate. J Appl Mech, September issue, 1957. 